\title{
El ser y el tener de los habitantes de la ciudad de Medellín como determinantes de la satisfacción con la vida
}

\section{To be and to have of the city of Medellin inhabitants as determinants of life satisfaction}

Catalina Gómez Toro Gabriel Jaime Suárez Obando Juan Esteban Garzón Trujillo Javier Alberto Gómez Gómez*

\section{Resumen}

El presente estudio tiene como objetivo encontrar y analizar los efectos causales de diversos factores socioeconómicos y demográficos en la satisfacción con la vida de los hogares de Medellín. Para ello, se estima un modelo logístico, categorizando en dos grupos las variables explicativas: los aspectos inherentes al ser y las características relacionadas al tener. Los principales resultados sugieren que estar soltero en relación con otro estado civil tiene un efecto negativo en el bienestar subjetivo, así como el hecho de ser afrodescendiente con respecto a otro grupo étnico. Por el lado del tener, cabe resaltar que mayores niveles de educación y estratos socioeconómicos más altos incrementan el bienestar subjetivo. Se concluye que tanto

* Centro de Investigaciones Económicas y Financieras (CIEF) - Universidad EAFIT, Carrera 497 Sur-50, Medellín, Colombia.

Contactos: Gómez Toro: cgomezt1@eafit.edu.co, Suárez Obando: gsuarez2@eafit.edu.co, Garzón Trujillo: jgarzon9@eafit.edu.co, Gómez Gómez: gomezjavieralberto@gmail.com.

Agradecemos a Lina Cardona Sosa, Marcelo Gantier Mita, al Comité Editorial Interno y al Comité Editorial Externo de la Revista LAJED, por los valiosos comentarios y sugerencias. 
las características del ser como las del tener son fundamentales para explicar la satisfacción con la vida, y por tanto, para la toma de decisiones de política pública.

Palabras clave: Satisfacción con la vida, modelo logístico, Medellín.

\begin{abstract}
This study aims to find and analyze the causal effects of various socioeconomic and demographic factors in life satisfaction of households in the city of Medellín. For this, a logistic model is estimated, categorizing into two groups the explanatory variables: the inherent aspects of being and the characteristics related with having material goods. The main results suggest that being single in relation to another marital state has a negative effect on subjective well being as well as being from African descent over another ethnic group. On the side of having, it should be noted that higher levels of education and higher socioeconomic strata increases subjective well being. It is concluded that both, the characteristics of "being" like those of "have" are fundamental to explain life satisfaction, and therefore for making public policy decisions.
\end{abstract}

Keywords: Life satisfaction, Logistic Model, Medellín.

\title{
Clasificación/Classification JEL: I31, C25
}

\section{Introducción}

La búsqueda de políticas públicas encaminadas a mejorar el bienestar de los habitantes de los países es una temática que cada vez toma mayor fuerza y relevancia, siendo un reto para los hacedores de políticas públicas; en esta temática la academia encuentra un espacio para aportar con sólida fundamentación teórico-empírica.

Inicialmente, los estudios enfocados en el bienestar subjetivo de las personas fueron abordados por filósofos, psicólogos, sociólogos y teólogos, tal como lo menciona Rojas (2009), constituyéndose luego en un campo de estudio para los economistas que buscaban un análisis sistemático de la felicidad mediante el relacionamiento de ésta con variables económicas como el ingreso, el desempleo y la inflación, entendiendo la felicidad como proxy de la utilidad del individuo. 
Para analizar los determinantes de la satisfacción con la vida de una determinada población, es indispensable partir de un concepto de satisfacción. En este sentido, podemos apoyarnos en autores como Veenhoven $(1994)^{1}$ que define la satisfacción como "el grado en que una persona evalúa la calidad global de su vida en conjunto de forma positiva” (4). En otras palabras, cuánto le gusta a una persona la vida que lleva (Beytía and Calvo, 2011, p. 2) al definirlo como "el grado con que una persona aprecia la totalidad de su vida presente de forma positiva y experimenta afectos de tipo placentero."

Dado lo anterior, se puede argumentar que el concepto de satisfacción con la vida se deriva del concepto de calidad de vida, con el cual se intenta definir qué es una buena vida, evaluar lo bien que se vive y averiguar qué se requiere para hacer la vida mejor, por lo que a lo largo de los años el concepto ha tenido variaciones, dependiendo del enfoque dado.

Así, la felicidad como área de estudio de la economía tomó especial sentido en la década de los años setenta, con los aportes de Easterlin (1974) y su paradoja que relaciona el ingreso y la felicidad que reportan las personas.

La temática alrededor del bienestar subjetivo de la población, el cual dentro de la literatura puede denominarse como el nivel de satisfacción con la vida o como felicidad, ha cobrado gran importancia, a tal punto que, como se argumenta en The World Happiness Report, publicado en los años 2012, 2013 y 2015, la medición del bienestar subjetivo de la población se ha convertido en un indicador para evaluar el progreso de las naciones, desplazando en cierta medida el producto interno bruto por habitante como proxy de calidad de vida (WHR, 2015).

Esta temática ha tenido tal impacto, que incluso países como Bután tienen un índice que mide la Felicidad Nacional Bruta, el cual es una filosofía y un instrumento de política que busca promover el desarrollo humano con una estrategia sostenible (Zurick, 2006). En cuanto a la medición, es importante resaltar que se establece con base en encuestas, lo que incorpora subjetividad en la respuesta del individuo. En las metodologías utilizadas se encuentran diferencias, ya que algunas encuestas simplemente preguntan a la persona si se encuentra o no satisfecha con la vida, mientras otras establecen categorías que varían entre muy satisfecho, satisfecho, poco satisfecho o insatisfecho; encontrando además mediciones ordinales y cardinales de la satisfacción con la vida.

1 Veehoven es sociólogo y el pionero en el estudio científico de la felicidad. Es director del World Data Base of Happiness en Holanda, dirigido por la Universidad de Rotterdam. 
Finalmente, puede decirse que un estudio que se acerca al que se pretende adelantar lo realizaron Medina y Tamayo (2012), quienes buscan encontrar cómo el crimen y la victimización afecta el nivel de satisfacción de la vida en Medellín. Sin embargo, esta propuesta se diferencia de la anterior en que no se pretende enfocar el análisis en la violencia, sino encontrar relaciones entre otras variables, como género, edad, raza, estado civil, número de hijos, nivel educativo, estrato socioeconómico, actividad productiva, tipo de vivienda, pensión y acceso a servicios públicos ${ }^{2}$. De manera innovadora, se clasifican estas variables en dos categorías: las propias al ser humano (género, raza, hijos, edad, etc.) y las que hacen referencia al tener (relacionadas al ingreso y estabilidad económica), lo cual puede llegar a aportar ideas propositivas de política pública que busquen mejorar la felicidad de los habitantes del municipio de Medellín.

En la primera parte del artículo se aborda la revisión de la literatura de las variables socioeconómicas que influyen en la satisfacción con la vida de los habitantes. Luego se procede con el modelo probabilístico, para encontrar relaciones entre variables seleccionadas a la luz de la teórica económica, de la Encuesta de Calidad de Vida (2014) con la probabilidad de que un individuo de la ciudad de Medellín se declare satisfecho con la vida. Más adelante se encuentra la sección que describe los resultados del modelo, para finalmente proceder con las conclusiones y recomendaciones.

\section{Revisión de la literatura}

El análisis económico del bienestar subjetivo ha sido abordado tanto desde el ámbito económico como desde una perspectiva social. Al respecto, Ansa Eceiza (2005) describe cómo la información para medir la felicidad se obtiene de cuestionarios donde se pregunta a los individuos sobre actividades de recreación, empleo, ingresos y seguridad en los barrios, entre otros, para buscar entender qué afecta verdaderamente el nivel de satisfacción en la vida.

Otros autores, como Di Tella, MacCulloch, y Oswald (2003), se han preocupado por estudiar los determinantes de la felicidad dado el ambiente macroeconómico de los países, encontrando que hay movimiento en el bienestar reportado por los individuos y que está correlacionado con cambios en variables macroeconómicas como el producto interno bruto, además que en países de Estado benefactor, mayores niveles de beneficios o ayudas al

2 También se destaca que el estudio de Medina y Tamayo es para el año 2012, mientras éste se realiza con la ECV 2014. 
desempleo están asociadas con un nivel mayor de bienestar nacional, así como la relación del bienestar subjetivo con el nivel de precios.

Otro enfoque para analizar el bienestar individual tiene que ver con el efecto de la capacidad institucional, la corrupción, el Estado de derecho, la calidad del gobierno y el tamaño del capital social, como lo argumentan Frey y Stutzer (2002a).

Por su parte Staubli, Killias y Frey (2014) analizan cómo el crimen afecta la satisfacción con la vida, mientras Di Tella y MacCulloch (2008) relacionan el tema con las horas de trabajo, la degradación del medio ambiente, la inflación, el desempleo y el crimen.

Se aprecia entonces cómo hay diferentes tendencias y relación de variables para el estudio de la satisfacción con la vida, encontrando, como García-Viniegras y González Benítez (2000), que hay dos metodologías predominantes para abordar la temática:

- La primera metodología se centra en variables objetivas externas a las personas, donde se trata de medir la calidad de vida utilizando preferiblemente indicadores de corte sociodemográfico. Entre los factores objetivos se encuentran entre los más recurridos los relacionados con la salud (como esperanza de vida, mortalidad infantil, nutrición y servicios de salud) con la educación (como el índice de alfabetismo y el acceso a los servicios educacionales), y con el valor de los ingresos per cápita, el producto nacional bruto y el índice de delincuencia, entre otros.

- La segunda metodología considera la calidad de vida como una dimensión subjetiva determinada por la valoración que hace la persona de su propia vida, metodología a partir de la cual se han realizado innumerables esfuerzos en la elaboración de técnicas e instrumentos que permitan la apreciación subjetiva y el nivel de satisfacción global o parcial en áreas importantes de la vida.

También se pueden encontrar visiones como la de Cruz y Torres (2006), que al citar a Blanchflower y Oswald (2000) explican que el ingreso no parece ser tan importante, pues los incrementos de felicidad obtenidos por mayores ingresos son pequeños y las variables no económicas aparecen como las más relevantes para explicarlos. También cuando al citar a Frey y Stutzer (2002) dicen que al comparar individuos encuentran que las personas con más ingreso tienen mayores niveles de felicidad; pero que, sin embargo, el ingreso tiene una utilidad marginal decreciente con respecto a la felicidad, es decir que el dinero compra felicidad pero no la hace crecer de manera infinita. 
Dentro de las características inherentes a las personas, podemos encontrar cómo Dolan, Peasgood y White (2008) encuentran que las mujeres tienden a reportar mayor felicidad que los hombres (Alesina, Di Tella y MacCulloch, 2004). Sin embargo, los estudios de Louis y Zhao (2002) reportan que no existen diferencias en cuanto a género, inclusive utilizando el mismo conjunto de datos. Dolan et al. (2008) sostienen que existe una correlación negativa entre la satisfacción con la vida y la edad, pero una correlación positiva entre la satisfacción con la vida y la edad al cuadrado. Esta relación aparece sistemáticamente en varios estudios, como los de Blanchflower y Oswald (2004) y Ferrer-i Carbonell y Gowdy (2007).

En lo que respecta a la raza o grupo étnico, en los Estados Unidos los blancos reportan mayor satisfacción con la vida que los afroamericanos (Thoits y Hewitt, 2001). Sin embargo, al comparar la raza blanca con la categoría “otros", es difícil hallar resultados, pues los resultados no son tan claros (Theodossiou, 1998); esto puede explicar por qué diferentes razas, como por ejemplo los latinos, reportan mayor satisfacción con la vida que los blancos. Así, los resultados de la comparación dependerán de los grupos raciales que contenga la categoría "otros" (Luttmer et al., 2005).

Blanchflower y Oswald (2004) encuentran que es peor para la satisfacción con la vida el hecho de encontrarse solo que en una relación, y si la relación se vuelve cada vez más estable, los efectos positivos se vuelven cada vez más fuertes. Lo que parece significar que el matrimonio o algo cercano a esto es positivo para la satisfacción con la vida. Sin embargo, los resultados varían dependiendo del estudio. El matrimonio entonces se asocia con el mayor nivel de satisfacción con la vida, mientras que el estar separado se asocia con el menor nivel de satisfacción con la vida, aun más que estar divorciado o viudo (Helliwell, 2003).

En lo concerniente a condiciones socioeconómicas, que se relacionan en mayor medida con la estabilidad en el ingreso que con las características de las personas, se encuentra que la relación positiva entre cada nivel adicional de educación y la satisfacción con la vida es clara a través de varios estudios, como Blanchflower y Oswald (2004); mientras tanto, otros estudios sugieren que la educación media está asociada al mayor nivel de satisfacción con la vida (Stutzer, 2004). Los diferentes estudios han mostrado una relación positiva entre el ingreso y la satisfacción con la vida; sin embargo, a medida que se aumenta el ingreso, el efecto es decreciente (Diener et al., 2002; Graham, Eggers y Sukhtankar, 2004; Marks y Fleming, 1999; Schyns, 2001). 
En lo relativo al empleo, se encuentran estudios que muestran una clara relación negativa entre el desempleo y la satisfacción con la vida. Para los modelos que miden esta temática como una variable continua, encuentran que el desempleo puede afectar negativamente entre un 5 y un 15\% los niveles de satisfacción con la vida (Di Tella et al., 2001; Frey y Stutzer, 2000; Frey y Stutzer, 2002b; Helliwell, 2003; Stutzer, 2004). Para el caso europeo, Lelkes (2006) encontró que el desempleo afecta negativamente la probabilidad de un alto nivel de satisfacción con la vida en un 19\%; y a la probabilidad de una alta felicidad, en un 15\%.

Finalmente, otra variable relacionada con condiciones socioeconómicas es el tipo de vivienda, lo cual, según Galay (2007) es esencial en los indicadores, encontrando para Bután que cuando las personas o familias no poseen casa propia, se ven forzadas a vivir en casas pequeñas o en casas superpobladas, lo que claramente y como lo muestra su estudio se traduce en menores niveles de satisfacción con la vida o felicidad. Además, la relación se hace igualmente positiva y más fuerte cuando se reporta que la vivienda es propia.

\section{Descripción de los datos}

Los datos utilizados en este estudio provienen de la Encuesta de Calidad de Vida de Medellín (ECVM) del año 2014, la cual posee información detallada de una muestra representativa de más de 13.000 hogares de la ciudad de Medellín, sobre los siguientes aspectos: situación laboral, ambiente familiar, características personales, condiciones de salud y vivienda, y percepción ciudadana. En la encuesta, al jefe de hogar se le pide calificar en una escala ordenada la calidad de vida de los miembros de su hogar con las siguientes opciones: "Muy buena", "Buena", "Aceptable", "Mala" y "Muy mala". Con base en el criterio de Medina y Tamayo (2012), los hogares cuya respuesta fue "Muy buena” y "Buena” son clasificados como satisfechos con la vida.

Los determinantes de la calidad de vida utilizados en este estudio son elegidos según la literatura encontrada, principalmente con base a las investigaciones de Dolan et al. (2008), Medina y Tamayo (2012) y Singer (2013). Una revisión de la literatura económica de los factores asociados con el bienestar subjetivo fue realizada por Dolan et al. (2008), quienes encuentran desde relaciones causales opuestas, para algunas variables, hasta consensos totales, en los efectos de otras. En específico, los autores analizan los artículos publicados en revistas de economía, investigaciones clave en el campo de la psicología y algunos working papers 
importantes sobre economía de la felicidad desde 1990. La evidencia empírica recopilada por ellos es un punto de referencia para el presente estudio, dada la globalidad de su investigación.

Medina y Tamayo (2012) también utilizan la ECVM del año 2008. Ellos analizan principalmente el impacto del crimen urbano y la victimización en la satisfacción con la vida, y su conclusión es que ambos factores tienen un efecto negativo y estadísticamente significativo. Si bien el resultado es interesante, el aporte para el presente estudio es la variedad de variables por las que controlan, pues, dado que provienen de la misma encuesta, aunque en diferente año, esto permite comparar la dirección de los efectos y sus magnitudes. Del mismo modo, Singer (2013) examina el impacto del soborno en la satisfacción con la vida en países de América Latina, y controla por factores demográficos relevantes para realizar una comparación.

Es fundamental establecer a priori los signos esperados de las variables explicativas con base en los datos disponibles. El Gráfico 1 muestra el porcentaje de satisfacción con la vida según el nivel de educación alcanzado. Se puede observar que, a mayor nivel de educación, mayor es el porcentaje de satisfechos y menor el de insatisfechos, es decir, se espera que la educación tenga un efecto positivo en la calidad de vida. La misma afirmación puede hacerse con respecto al estrato socioeconómico: entre más alto sea el estrato, más grande es la brecha entre satisfechos e insatisfechos (Gráfico 2).

A partir del Gráfico 3 es posible afirmar que las personas cuya ocupación es empleado, estudiante, rentista o jubilado/pensionado poseen los mayores porcentajes de satisfechos con la vida, mientras que aquellos desempleados o incapacitados permanentemente para trabajar tienen los mayores porcentajes de insatisfechos. Luego, se espera que las primeras categorías tengan un efecto positivo en relación con las últimas. 
Gráfico 1: Porcentaje de satisfechos e insatisfechos con la vida clasificados por nivel de educación

$120.00 \%$

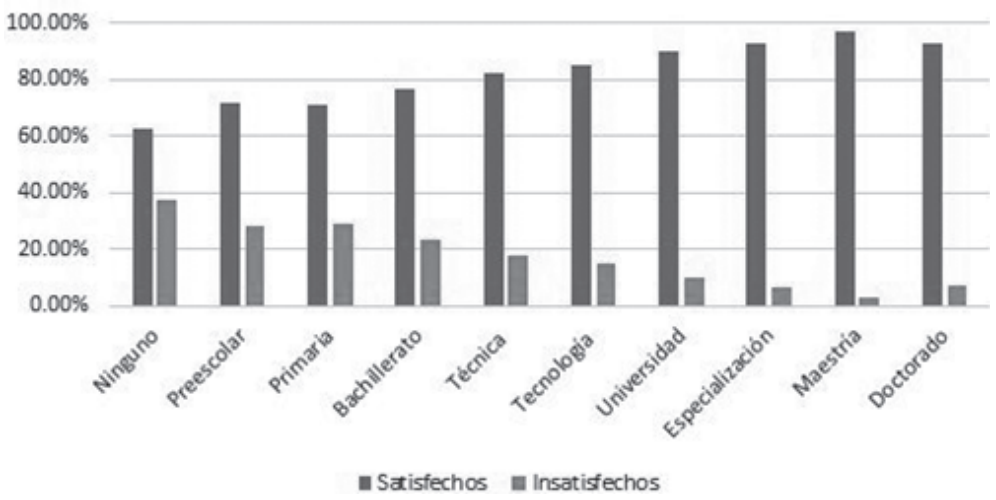

Gráfico 2: Porcentaje de satisfechos e insatisfechos con la vida clasificados por estrato socioeconómico

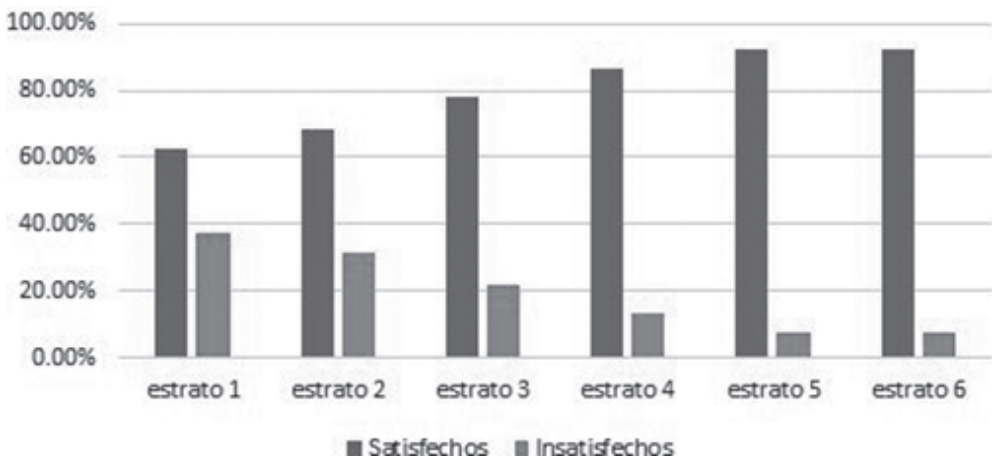




\section{Gráfico 3: Porcentaje de satisfechos e insatisfechos con la vida clasificados por ocupación}

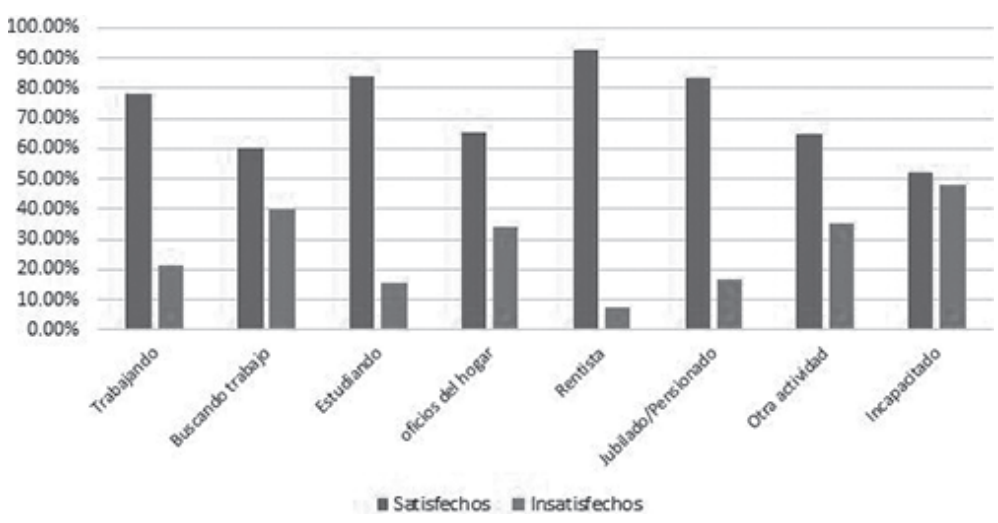

En el Gráfico 4 se puede observar que los solteros tienen el mayor porcentaje de insatisfechos con la vida. Esto es un indicio para esperar un efecto negativo de estar soltero con respecto a otro estado civil. Por otro lado, cabe resaltar que los hogares con vivienda propia totalmente pagada tienen la mayor proporción de satisfechos con la vida, según el Gráfico 5, por lo que se espera un efecto positivo en relación a aquellos hogares sin vivienda propia o en otro tipo de condición.

En cuanto a la percepción de la seguridad en el barrio, se espera que los hogares que se sienten inseguros tengan menor satisfacción con la vida que aquellos seguros o muy seguros, como se puede ver en el Gráfico 6, que muestra que los hogares inseguros tienen un porcentaje mayor de insatisfechos con la vida.

Los signos esperados de otras variables se muestran en el Cuadro 1. Este cuadro muestra los porcentajes de satisfechos e insatisfechos con la vida según los valores de las siguientes variables categóricas: género, grupo étnico, afiliación a pensiones, telefonía, gas natural, internet $y$ tv por suscripción. 
Gráfico 4: Porcentaje de satisfechos e insatisfechos con la vida clasificados por estado civil

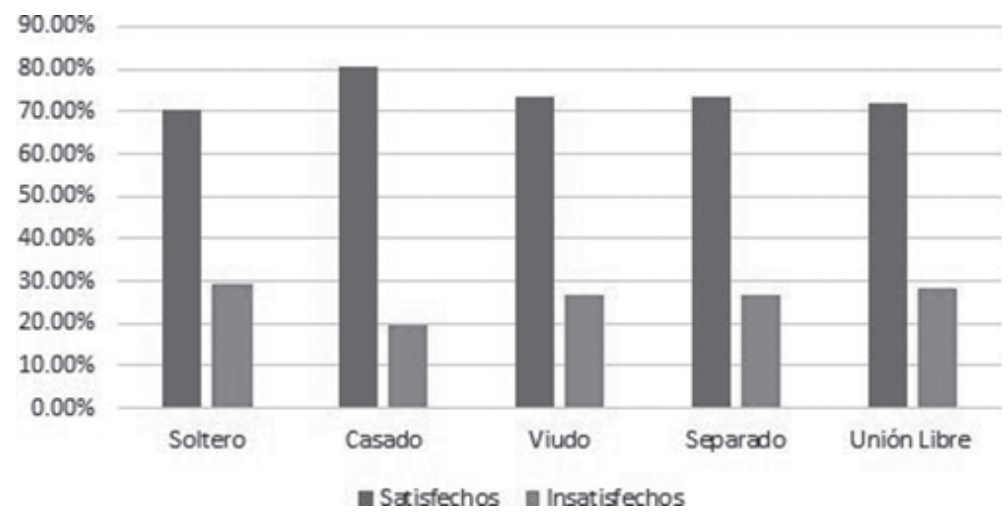

Gráfico 5: Porcentaje de satisfechos e insatisfechos con la vida clasificados por tipo de vivienda

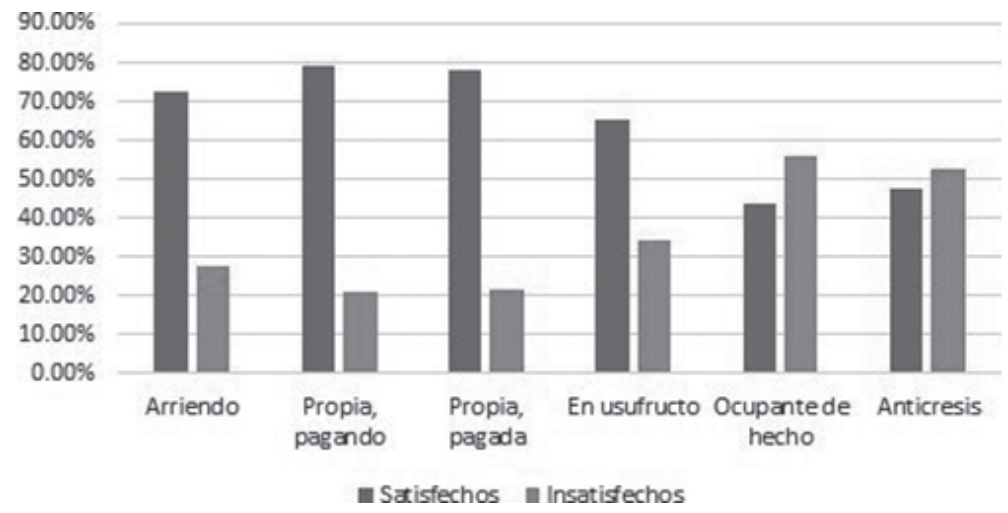


Gráfico 6: Porcentaje de satisfechos e insatisfechos con la vida clasificados por percepción de seguridad del barrio

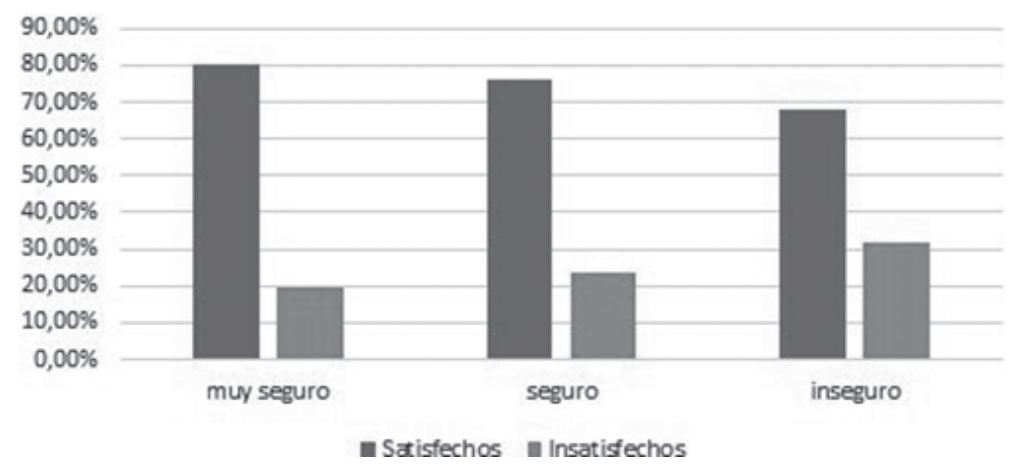

Cuadro 1

Porcentaje de satisfechos e insatisfechos con la vida por categorías de otras variables

\begin{tabular}{|c|c|c|c|}
\hline Variable & Satisfechos & Insatisfechos & Signo esperado \\
\hline \multicolumn{4}{|l|}{ Género } \\
\hline Masculino [base] & $79.01 \%$ & $20.99 \%$ & \multirow{2}{*}{-} \\
\hline Femenino & $71.22 \%$ & $28.78 \%$ & \\
\hline \multicolumn{4}{|l|}{ Grupo étnico } \\
\hline Otro [base] & $75.44 \%$ & $24.56 \%$ & \multirow[b]{2}{*}{ - } \\
\hline Afrodescendiente & $65.74 \%$ & $34.26 \%$ & \\
\hline \multicolumn{4}{|l|}{ Afiliación a Pensiones } \\
\hline No [base] & $69.99 \%$ & $30.01 \%$ & \multirow[b]{2}{*}{+} \\
\hline $\mathrm{Si}$ & $83.51 \%$ & $16.49 \%$ & \\
\hline \multicolumn{4}{|l|}{ Telefonía } \\
\hline No [base] & $60.85 \%$ & $39.15 \%$ & \multirow{2}{*}{+} \\
\hline $\mathrm{Si}$ & $77.47 \%$ & $22.53 \%$ & \\
\hline \multicolumn{4}{|l|}{ Gas Natural (Red) } \\
\hline No [base] & $77.22 \%$ & $22.78 \%$ & \multirow{2}{*}{+} \\
\hline $\mathrm{Si}$ & $67.80 \%$ & $32.20 \%$ & \\
\hline \multicolumn{4}{|l|}{ Gas Natural } \\
\hline No [base] & $67.04 \%$ & $32.96 \%$ & \multirow[b]{2}{*}{+} \\
\hline $\mathrm{Si}$ & $78.32 \%$ & $21.68 \%$ & \\
\hline \multicolumn{4}{|l|}{ Internet } \\
\hline No [base] & $64.86 \%$ & $35.14 \%$ & \multirow{2}{*}{+} \\
\hline $\mathrm{Si}$ & $83.00 \%$ & $17.00 \%$ & \\
\hline \multicolumn{4}{|l|}{ TV por suscripción } \\
\hline No [base] & $60.14 \%$ & $39.86 \%$ & \multirow{2}{*}{+} \\
\hline $\mathrm{Si}$ & $80.30 \%$ & $19.70 \%$ & \\
\hline
\end{tabular}


El Cuadro 2 contiene la estadística descriptiva de las variables clasificadas por satisfacción con la vida. El tamaño de la muestra obtenida, después de eliminar los valores faltantes y algunos datos atípicos, es de 12,575 hogares, de los cuales el 25\% se consideran con baja calidad de vida. Las variables que hacen referencia a características personales recogen información únicamente sobre el jefe del hogar encuestado, debido a que éstas son de vital importancia para la calidad de vida de todos los miembros de su hogar.

Cuadro 2

Estadística descriptiva

\begin{tabular}{|c|c|c|c|c|}
\hline \multirow[b]{2}{*}{ Variable } & \multicolumn{2}{|c|}{ Insatisfechos } & \multicolumn{2}{|c|}{ Satisfechos } \\
\hline & Media & Desv. Est. & Media & Desv. Est. \\
\hline \multicolumn{5}{|l|}{ Educación alcanzada } \\
\hline Ninguno [base] & 0.234 & 0.423 & 0.131 & 0.337 \\
\hline Preescolar & 0.008 & 0.089 & 0.007 & 0.082 \\
\hline Primaria & 0.351 & 0.477 & 0.286 & 0.452 \\
\hline Bachillerato & 0.302 & 0.459 & 0.331 & 0.47 \\
\hline Técnica & 0.041 & 0.198 & 0.062 & 0.242 \\
\hline Tecnología & 0.022 & 0.146 & 0.041 & 0.198 \\
\hline Universidad & 0.035 & 0.184 & 0.104 & 0.305 \\
\hline Especialización & 0.006 & 0.076 & 0.026 & 0.16 \\
\hline Maestría & 0.001 & 0.031 & 0.01 & 0.098 \\
\hline Doctorado & 0.001 & 0.025 & 0.003 & 0.051 \\
\hline \multicolumn{5}{|l|}{ Estado civil } \\
\hline Soltero [base] & 0.252 & 0.434 & 0.199 & 0.4 \\
\hline Casado & 0.285 & 0.451 & 0.391 & 0.488 \\
\hline Viudo & 0.136 & 0.342 & 0.125 & 0.33 \\
\hline Separado & 0.111 & 0.314 & 0.1 & 0.3 \\
\hline Unión Libre & 0.217 & 0.412 & 0.185 & 0.388 \\
\hline \multicolumn{5}{|l|}{ Estrato socioeconómico } \\
\hline 1 [base] & 0.191 & 0.393 & 0.106 & 0,308 \\
\hline 2 & 0.453 & 0.498 & 0.323 & 0.468 \\
\hline 3 & 0.262 & 0.44 & 0.308 & 0.462 \\
\hline 4 & 0.06 & 0.237 & 0.127 & 0.333 \\
\hline 5 & 0.023 & 0.151 & 0.092 & 0.289 \\
\hline 6 & 0.011 & 0.102 & 0.043 & 0.204 \\
\hline \multicolumn{5}{|l|}{ Tipo de vivienda } \\
\hline Arriendo & 0,395 & 0.489 & 0.346 & 0.476 \\
\hline Propia, parcialmente pagada & 0,045 & 0.207 & 0.056 & 0.23 \\
\hline Propia, totalmente pagada [base] & 0.444 & 0.497 & 0.53 & 0.499 \\
\hline En usufructo & 0.1 & 0.301 & 0.063 & 0.243 \\
\hline Ocupante de hecho & 0.013 & 0.114 & 0.003 & 0.058 \\
\hline Anticresis & 0.003 & 0.056 & 0.001 & 0.031 \\
\hline
\end{tabular}




\begin{tabular}{lcccc}
\hline & \multicolumn{2}{c}{ Insatisfechos } & \multicolumn{2}{c}{ Satisfechos } \\
\hline \multicolumn{1}{c}{ Variable } & Media & Desv. Est. & Media & Desv. Est. \\
\hline Seguridad barrio & & & & \\
$\quad$ Muy seguro & 0.05 & 0.218 & 0.067 & 0.25 \\
$\quad$ Seguro & 0.754 & 0.431 & 0.795 & 0.404 \\
$\quad$ Inseguro [base] & 0.196 & 0.397 & 0.138 & 0.345 \\
Ocupación & & & & \\
$\quad$ Trabajando & 0.45 & 0.498 & 0.541 & 0.498 \\
$\quad$ Buscando trabajo [base] & 0.044 & 0.205 & 0.022 & 0.146 \\
$\quad$ Estudiando & 0.007 & 0.084 & 0.012 & 0.111 \\
$\quad$ Oficios del hogar & 0.31 & 0.463 & 0.196 & 0.397 \\
$\quad$ Rentista & 0.001 & 0,025 & 0.003 & 0.051 \\
$\quad$ Jubilado/Pensionado & 0.11 & 0,313 & 0.184 & 0.387 \\
$\quad$ Otra Actividad & 0.057 & 0,231 & 0.035 & 0.183 \\
$\quad$ Incapacitado para trabajar & 0.021 & 0,145 & 0.008 & 0.088 \\
Mujer & 0.576 & 0.494 & 0.472 & 0.499 \\
Edad & 52494 & 15757 & 52480 & 16116 \\
Negro & 0.043 & 0.203 & 0.027 & 0.163 \\
Niños en el hogar & 0.602 & 0.952 & 0.491 & 0.81 \\
Afiliación a Pensiones & 0.252 & 0.434 & 0.423 & 0.494 \\
Telefonía & 0.779 & 0.415 & 0.886 & 0.317 \\
Gas Natural (Red) & 0.626 & 0.484 & 0.748 & 0.434 \\
Gas Natural & 0.287 & 0.452 & 0.2 & 0.4 \\
Internet & 0,387 & 0.487 & 0.626 & 0.484 \\
TV por suscripción & 0,589 & 0.492 & 0.795 & 0.404 \\
Ingreso familiart & 1236998 & 1095084 & 2021248 & 1728919 \\
\hline N & $\mathbf{3 1 2 7}$ & & $\mathbf{9 4 4 8}$ \\
\hline
\end{tabular}

† El ingreso familiar se estandariza al incluirse en el modelo

\subsection{Características inherentes al individuo}

Del cuadro anterior cabe resaltar que aproximadamente el $24 \%$ de los hogares insatisfechos no posee algún nivel de educación, el 65\% alcanzó primaria o bachillerato y sólo el 10\% alcanzó un nivel de educación superior. Los porcentajes respectivos de los hogares satisfechos son $13.1 \%, 61.7 \%$ y $24.5 \%$, lo que indica que éstos en general poseen, proporcionalmente, mayores niveles de educación que los hogares insatisfechos. Por otro lado, se tiene que el $28.5 \%$ de los jefes de hogar insatisfechos están casados, seguido de los solteros (25.2\%) y los que tienen una unión libre $(21.7 \%)$. En relación a los satisfechos, es superior el porcentaje de casados (39.1\%) y menor el de solteros (20\%) y en unión libre (18.5\%). 
Se puede observar que la edad promedio de los jefes de hogar en ambos grupos es de 52 años, aproximadamente; además, la proporción de mujeres de los hogares satisfechos (47.2\%) es menor que aquélla de los hogares insatisfechos (57.6\%). Por otro lado, como es de esperarse, puede verse que en los hogares insatisfechos viven en promedio más niños, la proporción de afrodescendientes es mayor y se consideran más inseguros en su barrio.

\subsection{Características externas}

Como se indica en el Cuadro 2, el $58.6 \%$ de los hogares satisfechos posee vivienda propia, y el $34.6 \%$ se encuentra pagando arriendo. En comparación, el $48.9 \%$ de los hogares insatisfechos tiene vivienda propia y el 39.5\% pagan arriendo. Respecto al estrato socioeconómico, de los hogares satisfechos, el $42.9 \%$ pertenece a los estratos 1 o 2, 43.5\% a los estratos 3 o 4, y el 13.5\% viven en los estratos 5 o 6. Por el lado de los hogares insatisfechos, es mayor el porcentaje de hogares en estratos 1 o $2(64.4 \%)$ y menor el de estratos 3 o 4 (32.2\%) y 5 o $6(3.4 \%)$.

Adicionalmente, cabe resaltar que los hogares satisfechos tienen mayor acceso a servicios públicos como telefonía, internet, TV por suscripción y red de gas natural, que los insatisfechos. Por último, nótese que hay un mayor porcentaje de jefes de hogares satisfechos que se encuentran trabajando o están jubilados/pensionados, mientras que en los hogares insatisfechos el porcentaje de jefes que están buscando trabajo o se dedican a oficios del hogar es significativamente mayor.

\section{Metodología}

Para la estimación de la probabilidad que los individuos se declaren satisfechos con la vida, se estima un modelo de elección discreta en donde la variable dependiente es dicotómica e indica si un individuo se siente satisfecho con su vida o no. Los datos de corte transversal son elegidos teniendo en consideración los resultados más relevantes para la toma de decisiones de política pública, por eso se emplea la ECVM del año 2014.

Sea $y_{i}^{*}$, no observable, el nivel subjetivo de satisfacción con la vida del individuo $i$, determinado por la siguiente ecuación:

$$
y_{i}^{*}=x_{i}^{\prime} \beta+\varepsilon_{i} \text { para } i=1, \ldots, N
$$


donde $x_{i}$ es el vector $K \times 1$ de covariados, $\beta$ es el vector $K \times 1$ de parámetros y $\varepsilon_{i}$ es el error idiosincrático del individuo $i$. Luego, la variable observada es $y_{i}$, definida de la siguiente manera:

$$
y_{i}=\left\{\begin{array}{l}
1 \text { si } y_{i}^{*}>0 \\
0 \text { si } y_{i}^{*} \leq 0
\end{array}\right.
$$

ahora, la probabilidad de que un individuo se considere satisfecho con su vida está determinada por la siguiente expresión:

$$
\begin{gathered}
P\left(y_{i}=1 \mid x\right)=P\left(y_{i}^{*}>0\right) \\
=P\left(x_{i} \beta+\varepsilon_{i}>0\right) \\
=P\left(\varepsilon_{i}>-x_{i} \beta\right) \\
=1-P\left(\varepsilon_{i} \leq-x_{i} \beta\right) \\
=1-\Lambda\left(x_{i} \beta\right) \\
P\left(y_{i}=1 \mid x\right)=\Lambda\left(x_{i} \beta\right)=\frac{e^{x_{i} \beta}}{1+e^{x_{i} \beta}}
\end{gathered}
$$

El método de estimación de los parámetros $\beta$ del modelo de elección discreta utilizado (Logit) se denomina "Máxima verosimilitud", y consiste en estimar los valores de los parámetros que maximizan la función de densidad conjunta (verosimilitud) o probabilidad de obtener la muestra observada. Se parte de la función de distribución de los datos, y generalmente se consideran las funciones logística y normal estándar. Luego se construye la función de verosimilitud, y finalmente se hallan los parámetros mediante su optimización (Cameron y Trivedi, 2005).

Dado lo anterior, calcular el efecto que un cambio en la variable $x_{j}$ produce en la ecuación (3) resulta de las siguientes expresiones, para variables continuas y discretas, respectivamente:

$$
\frac{\partial P\left(y_{i}=1 \mid x\right)}{\partial x_{j}}=\Lambda\left(x_{i} \beta\right)\left[1-\Lambda\left(x_{i} \beta\right)\right] \beta_{j}
$$




$$
\frac{\Delta P\left(y_{i}=1 \mid x\right)}{\Delta x_{j}}=\Lambda\left(x_{1} \beta_{1}+\ldots+\left(x_{j}=1\right) \beta_{j}+\ldots+x_{k} \beta_{k}\right)-\Lambda\left(x_{1} \beta_{1}+\ldots+0+\ldots+x_{k} \beta_{k}\right)
$$

En la práctica, las ecuaciones (4) y (5) se estiman de dos formas diferentes, según los valores de $x$. Por un lado, se puede evaluar el efecto parcial en los promedios de los regresores $\left(\bar{x}_{1}, \ldots, \bar{x}_{k}\right)$, pero esto genera un problema, dado que el individuo medio no es representativo debido a la existencia de variables categóricas. Por otro lado, se puede evaluar el efecto parcial para cada individuo $i$, y posteriormente calcular el promedio de todos los efectos. Este enfoque, denominado "Efecto promedio marginal" (Average Marginal Effect) es considerado más apropiado, teniendo en cuenta que varias variables utilizadas en este estudio son categóricas.

El Average Marginal Effect (AME) está determinado por las siguientes ecuaciones para variables continuas y dicotómicas, respectivamente:

$$
\begin{gathered}
A M E_{k}=\beta_{k} \frac{1}{N} \sum_{i=1}^{N}\left[\Lambda\left(x_{i} \beta\right)\right] \\
A M E_{k}=\frac{1}{N} \sum_{i=1}^{N}\left[\Lambda\left(x_{i} \beta+\beta_{k}\right)-\Lambda\left(x_{i} \beta\right)\right]
\end{gathered}
$$




\section{Resultados}

\section{Cuadro 3 \\ Resultados Logit}

\begin{tabular}{|c|c|c|c|c|c|c|}
\hline Variable & Coeficiente & & (Error est.) & AME & & (Error est.) \\
\hline Mujer & -0.1600 & $* * *$ & $(0.062)$ & -0.0258 & $* * *$ & $(0.010)$ \\
\hline Edad & -0.0490 & $* * *$ & $(0.009)$ & -0.0016 & $* * *$ & $(0.0004)$ \\
\hline Edad al cuadrado & 0.0004 & $* * *$ & $(0.0001)$ & & & \\
\hline Negro & -0.2860 & $* *$ & $(0.118)$ & -0.0462 & $* *$ & $(0.019)$ \\
\hline \multicolumn{7}{|l|}{ Estado civil } \\
\hline Casado & 0.2320 & $* * *$ & $(0.071)$ & 0.0383 & $* * *$ & $(0.012)$ \\
\hline Viudo & 0.2270 & $* * *$ & $(0.085)$ & 0.0376 & $* * *$ & $(0.014)$ \\
\hline Separado & 0.2270 & $* * *$ & $(0.083)$ & 0.0376 & $* * *$ & $(0.014)$ \\
\hline Unión Libre & 0.1390 & $*$ & $(0.074)$ & 0.0233 & $*$ & $(0.012)$ \\
\hline \multicolumn{7}{|l|}{ Educación alcanzada } \\
\hline Preescolar & 0.4330 & * & $(0.257)$ & 0.0688 & * & $(0.037)$ \\
\hline Primaria & 0.1540 & $* *$ & $(0.064)$ & 0.0258 & $* *$ & $(0.011)$ \\
\hline Secundaria & 0.1500 & $* *$ & $(0.074)$ & 0.0251 & $* *$ & $(0.013)$ \\
\hline Técnica & 0.3250 & $* * *$ & $(0.123)$ & 0.0529 & $* * *$ & $(0.019)$ \\
\hline Tecnología & 0.2650 & * & $(0.154)$ & 0.0437 & $*$ & $(0.024)$ \\
\hline Universidad & 0.2720 & $* *$ & $(0.133)$ & 0.0446 & $* *$ & $(0.021)$ \\
\hline Especialización & 0.4820 & $*$ & $(0.267)$ & 0.0758 & $* *$ & $(0.038)$ \\
\hline Maestría & 1.1920 & $* *$ & $(0.599)$ & 0.1587 & $* * *$ & $(0.057)$ \\
\hline Doctorado & 0.2740 & & $(0.773)$ & 0.0449 & & $(0.120)$ \\
\hline Niños en el hogar & -0.1350 & $* * *$ & $(0.030)$ & -0.0219 & $* * *$ & $(0.005)$ \\
\hline \multicolumn{7}{|l|}{ Estrato socioeconómico } \\
\hline 2 & -0.0410 & $* * *$ & $(0.070)$ & -0.0072 & $* * *$ & $(0.012)$ \\
\hline 3 & 0.2510 & $* * *$ & $(0.080)$ & 0.0422 & $* * *$ & $(0.014)$ \\
\hline 4 & 0.5230 & $* * *$ & $(0.114)$ & 0.0829 & $* * *$ & $(0.018)$ \\
\hline 5 & 0.8900 & $* * *$ & $(0.154)$ & 0.1294 & $* * *$ & $(0.020)$ \\
\hline 6 & 0.6890 & $* * *$ & $(0.212)$ & 0.1052 & $* * *$ & $(0.029)$ \\
\hline Ingreso familiar & 0.4700 & $* * *$ & $(0.046)$ & 0.0845 & & $(0.008)$ \\
\hline Ingreso familiar al cuadrado & -0.0950 & & $(0.017)$ & & & \\
\hline \multicolumn{7}{|l|}{ Ocupación } \\
\hline Trabajando & 0.4410 & $* * *$ & $(0.124)$ & 0.0759 & $* * *$ & $(0.023)$ \\
\hline Estudiando & 0.5530 & $* *$ & $(0.275)$ & 0.0931 & $* *$ & $(0.043)$ \\
\hline Oficios del hogar & 0.2160 & $*$ & $(0.131)$ & 0.0387 & & $(0.024)$ \\
\hline Rentista & 1.6750 & $* *$ & $(0.765)$ & 0.2187 & $* * *$ & $(0.062)$ \\
\hline Jubilado/Pensionado & 0.5030 & $* * *$ & $(0.141)$ & 0.0855 & $* * *$ & $(0.025)$ \\
\hline Otra actividad & -0.0770 & & $(0.159)$ & -0.0144 & & $(0.030)$ \\
\hline Incapacitado para trabajar & -0.5390 & $* *$ & $(0.220)$ & -0.1065 & $* *$ & $(0.044)$ \\
\hline \multicolumn{7}{|l|}{ Tipo de vivienda } \\
\hline Arriendo & -0.3520 & $* * *$ & $(0.053)$ & -0.0572 & $* * *$ & $(0.009)$ \\
\hline
\end{tabular}




\begin{tabular}{|c|c|c|c|c|c|c|}
\hline Variable & Coeficiente & & (Error est.) & AME & & (Error est.) \\
\hline Propia, parcialmente pagada & -0.2560 & $* *$ & $(0.108)$ & -0.0408 & ** & $(0.018)$ \\
\hline En usufructo & -0.3950 & $* * *$ & $(0.082)$ & -0.0647 & $* * *$ & $(0.014)$ \\
\hline Ocupante de hecho & -0.8030 & $* * *$ & $(0.254)$ & -0.1408 & $* * *$ & $(0.050)$ \\
\hline Anticresis & -0.8830 & * & $(0.486)$ & -0.1565 & & $(0.097)$ \\
\hline Afiliación a Pensiones & 0.3080 & $* * *$ & $(0.057)$ & 0.0499 & $* * *$ & $(0.009)$ \\
\hline Telefonía & 0.1650 & $* *$ & $(0.065)$ & 0.0267 & $* *$ & $(0.011)$ \\
\hline Gas Natural (Red) & 0.2430 & $* * *$ & $(0.084)$ & 0.0393 & $* * *$ & $(0.014)$ \\
\hline Internet & 0.3270 & $* * *$ & $(0.053)$ & 0.0530 & $* * *$ & $(0.008)$ \\
\hline Gas natural & 0.2270 & $* *$ & $(0.090)$ & 0.0367 & $* *$ & $(0.014)$ \\
\hline TV por suscripción & 0.3740 & $* * *$ & $(0.053)$ & 0.0606 & $* * *$ & $(0.008)$ \\
\hline Seguridad barrio & & & & & & \\
\hline Muy seguro & 0.8220 & $* * *$ & $(0.110)$ & 0.1381 & $* * *$ & $(0.017)$ \\
\hline Seguro & 0.5820 & $* * *$ & $(0.060)$ & 0.1020 & $* * *$ & $(0.011)$ \\
\hline Constante & 0.8320 & $* * *$ & $(0.299)$ & & & \\
\hline $\mathbf{N}$ & \multicolumn{6}{|c|}{12575} \\
\hline Log-likelihood & \multicolumn{6}{|l|}{-6201.9510} \\
\hline Pseudo R squared & \multicolumn{6}{|l|}{0.1207} \\
\hline$\chi_{45}^{2}$ & \multicolumn{6}{|l|}{1701.9040} \\
\hline Prob & \multicolumn{6}{|l|}{0.0000} \\
\hline
\end{tabular}

Niveles de significancia:

$*: 10 \%$

$* *: 5 \%$

$* * *: 1 \%$

Del Cuadro 3 es posible inferir, en relación a las características inherentes al individuo (el ser), que se espera que el hecho de ser mujer disminuya la probabilidad de sentirse satisfecho con la vida en $2.5 \%$ con respecto a ser hombre. Si bien difiere con la evidencia empírica de otros estudios, es acorde con resultados encontrados para América Latina (Singer, 2013) y Colombia (Medina y Tamayo, 2012). En relación con la raza, se encuentra que ser afrodescendiente tiene un efecto negativo en la felicidad de $4.62 \%$ con respecto a cualquier otro grupo étnico, tal como sugiere la literatura. Esto puede explicarse por la discriminación social a la que se ve enfrentado dicho grupo étnico.

Como se ve en la literatura, el efecto de la edad resulta tener una forma de $\mathrm{U}$, por el coeficiente negativo de la edad y el coeficiente positivo de la edad al cuadrado. Es decir, en la juventud se experimenta un alto nivel de satisfacción con la vida, que va disminuyendo a medida que transcurre el tiempo, hasta alcanzar un mínimo en la etapa adulta. Posteriormente, vuelve a aumentar alllegar la vejez, cuando se alcanza de nuevo un alto nivel de satisfacción. Este resultado es bastante intuitivo, pues, generalmente, en la juventud se posee tanto el respaldo 
económico de los padres como gran cantidad de tiempo libre para el ocio y para recrearse con amistades, aspectos que aportan considerablemente a la felicidad, mientras que en la etapa adulta existen más responsabilidades, principalmente por la carga laboral, el cuidado de los hijos (en caso de tenerlos) y la necesidad de obtener ingresos para cubrir los gastos, entre otros aspectos que pueden generan preocupación, estrés, fatiga, etc., y en consecuencia disminuir el bienestar subjetivo. Luego, en la vejez, varias de las obligaciones de la etapa adulta desaparecen, como por ejemplo la carga laboral (jubilación o pensión) y el cuidado directo de los hijos, por lo cual la satisfacción se vuelve a incrementar.

En relación al estado civil del jefe de hogar, se encuentra que ser soltero disminuye la probabilidad de sentirse satisfecho con la vida en 3.83\% con respecto a estar casado, 3.76\% con respecto a ser viudo o separado y $2.33 \%$ en relación a estar en unión libre. Estos resultados son acordes con Medina y Tamayo (2012), que encuentran que ser soltero tiene un efecto negativo comparado con ser viudo.

Pasando a analizar las variables que se pueden categorizar dentro del tener, se encuentra cómo la educación del jefe de hogar tiene una relación positiva con la satisfacción con la vida. Por ejemplo, tener una técnica tiene un incremento de $5.28 \%$ con respecto a no tener algún nivel de educación; la magnitud aumenta para un nivel de especialización (7.57 \%) y maestría ( $15.89 \%)$; no obstante, el efecto es menor con una tecnología ( $4.34 \%$ ) o un título universitario (4.46\%) en relación con el mencionado para una técnica. Se observa un resultado similar con el estrato socioeconómico, en particular, residir en una vivienda de estrato 3 en adelante aumenta la probabilidad de sentirse satisfecho con la vida en relación a los estratos 1 y 2 . Pero cabe resaltar que la magnitud no es proporcional al estrato, por ejemplo, pertenecer al estrato 5 tiene un efecto menor que el estrato 4, y el estrato 6 un efecto menor que los anteriores. Adicionalmente, pertenecer al estrato 2 disminuye en $0.7 \%$ la probabilidad de satisfacción con la vida respecto al estrato 1 .

El efecto de la situación laboral varía con las categorías: en general, se observa que ser rentista tiene el mayor efecto (positivo) en la calidad de vida, de $21.87 \%$ en relación con estar desempleado. Trabajar la incrementa en $7.59 \%$ y estudiar en $9.31 \%$, estar jubilado o pensionado también tiene un efecto significativo de $8.54 \%$. Se encuentra además que estar en condición de discapacidad es la única ocupación -estadísticamente significativa- que tiene un efecto negativo; específicamente, ésta disminuye en 10.65\% la probabilidad de sentirse satisfecho con la vida con respecto a los desempleados. Tener otra ocupación (otra actividad) 
no es estadísticamente significativo, como es de esperarse, ya que puede existir una gran diferencia entre las ocupaciones no observadas.

De otro lado, se observa que tener una vivienda propia (totalmente pagada) es preferible a cualquier otra condición de tipo de vivienda; le sigue en orden de preferencia tener una vivienda propia (parcialmente pagada) y tener una vivienda arrendada.

Finalmente, estar afiliado a un sistema de pensiones aumenta en promedio 5\% la probabilidad de tener bienestar subjetivo. Igualmente, el acceso a servicios públicos y de entretenimiento también tiene un efecto positivo en la felicidad, ya que aumenta la utilidad de los individuos.

\section{Conclusiones}

El estudio de la felicidad, la satisfacción con la vida o el bienestar subjetivo de los individuos, es un tema que desde la época aristotélica ha sido estudiado, dado que el fin mismo del ser humano es encontrar su propia felicidad, así sea bajo las restricciones propias de cada individuo. Es así como la teoría italiana de la economía civil retoma estos fundamentos filosóficos, y los describe en el capítulo siete del Reporte Mundial de Felicidad (2015), en el que Becchetti, Bruni y Zamagni (2015) resaltan la importancia del trabajo mancomunado entre las instituciones y el mercado, junto con la responsabilidad social de los ciudadanos y de las corporaciones, en donde la familia, la confianza, la amistad y en general los valores humanos toman un papel protagónico.

De esta manera, el egoísmo del homo economicus, que solo busca maximizar su propio bienestar, se pone a prueba, y se piensa cómo se puede generar una cultura de la búsqueda del bienestar colectivo o de la comunidad. Este artículo aporta en este sentido desde dos perspectivas, las cuales, aunque parecen diferentes, se complementan: las propias del ser y las que hacen referencia al tener bienes materiales.

Dado que la experiencia de los países mostró con el tiempo que no sólo los ingresos de las personas influencian la capacidad de sentirse feliz, este hecho motivó a los países y ciudades a gestionar indicadores, buscando estimar el nivel de satisfacción con la vida y trabajar con las herramientas de la política pública para procurar mejores niveles de bienestar en la población. 
Se percibe entonces la importancia de aspectos diferentes a los económicos o monetarios, como clave para entender el sentimiento de bienestar de las personas. Este argumento se evidenció luego de la Segunda Guerra Mundial, cuando países como Estados Unidos, Japón y Reino Unido, entre muchos otros, evidenciaron un aumento pronunciado de su ingreso per cápita, pero el resultado de las encuestas que medían la percepción de satisfacción con la vida de los habitantes era constante o incluso disminuía un poco. Es por esto que se genera una motivación para agrupar los determinantes del bienestar subjetivo en dos categorías: las propias de los seres humanos y las que se relacionan directamente con los recursos monetarios de los individuos.

De esta manera, este estudio, aplicado al caso de la ciudad de Medellín, encuentra evidencia que aporta a otras investigaciones empíricas, y por ende a la literatura existente de economía de la felicidad. Tanto los aspectos relacionados con la estabilidad económica como las características propias de cada individuo inciden sobre la probabilidad de que la persona se declare satisfecha con la vida. Es relevante resaltar algunos elementos que se pueden mejorar de una u otra forma desde la política pública: acceso a mayores años de escolaridad, mejora en la calidad en la misma, disminución de la deserción escolar; esto de una forma se transformará en mejores posibilidades de empleo formal e ingresos futuros estables. Este aspecto resulta fundamental para los países latinoamericanos, en los que alrededor del cincuenta por ciento de la población trabajadora es informal, con las consecuencias en acceso a la seguridad social y presiones fiscales que esto implica.

En la política pública también recae la responsabilidad de buscar medios para que la población tenga acceso a servicios públicos universales y las oportunidades de obtener una vivienda propia, debido a que, dadas las restricciones económicas de los estratos socioeconómicos bajos, es difícil acceder a éstos con recursos propios, si sólo los ofrecen los privados.

Desde otra perspectiva, la falta de oportunidades para personas afrodescendientes, discapacitados para trabajar, minorías étnicas y diferencia de género, genera una disminución en la probabilidad de sentirse satisfecho con su vida. Al respecto, podría pensarse en programas direccionados a estas minorías, que propicien capacidades productivas, de empleo, de esparcimiento y no discriminatorias, que de una u otra manera mejoren su bienestar subjetivo y propicien mejoras en equidad que perduren en el tiempo. 
Es importante también señalar que, para la ciudad de Medellín, así como otras de Centro y Sudamérica, un aspecto que no se puede desconocer y que resulta protagonista es la seguridad. Al respecto, se encuentra en la literatura relacionada con la satisfacción con la vida, que la seguridad en los barrios de la ciudad es un factor explicativo significativo del bienestar subjetivo de las personas. De manera concreta, si bien la ciudad ha avanzado en la reducción de los indicadores de violencia, aún existen falencias caracterizadas por células vigentes del antiguo narcotráfico, así como por la existencia de bandas criminales urbanas, siendo el hurto, la extorsión, las lesiones personales y el microtráfico, entre otros, delitos contra los que hay que luchar asignando recursos eficientemente de la mano de innovaciones tecnológicas, así como el fortalecimiento disuasivo de la fuerza pública y el sistema judicial, con miras a garantizar la tranquilidad a las familias y por ende su bienestar.

Finalmente, en línea con la idea anterior, la firma del acuerdo de paz con el grupo guerrillero FARC (Fuerzas Armadas Revolucionarias de Colombia), y tal vez más adelante con el ELN (Ejército de Liberación Nacional), traerá el reto de reinsertar a la sociedad civil miles de ex combatientes y familias que con la paz podrían mejorar sus condiciones de vida. Sin embargo, muchos de estos ciudadanos están en las filas de los grupos guerrilleros desde la infancia, sin oportunidad de obtener un título bachiller, por lo que en realidad es un gran reto para los hacedores de política y para el sector privado, quienes deben procurar lograr lo que propone la economía civil italiana sobre las dos manos adicionales a las instituciones formales y el mercado: la responsabilidad social de las personas y de las empresas, para lograr un país en verdadera paz y prosperidad.

Fecha de recepción: 23 de agosto de 2016. Fecha de aceptación: 26 de septiembre de 2016. Manejado por la A.B.C.E. 


\section{Referencias}

1. Alesina, A., R. Di Tella, y R. MacCulloch. 2004. "Inequality and Happiness: are Europeans and Americans different?”. Journal of Public Economics, 88(9):2009-2042.

2. Ansa Eceiza, M. M. 2005. "Economía y felicidad: Acerca de la relación entre bienestar material y bienestar subjetivo". XI Jornadas de economía crítica. Bilbao, España.

3. Becchetti, L., L. Bruni y S. Zamagni. 2015. "Human values, civil economy, and subjective well-being". World happiness report 2015, págs. 132-151. New York

4. Beytía, P. y E. Calvo. 2011. ¿Cómo medir la felicidad? (How to measure happiness?). Claves de politicas públicas, 4, Institute at Universidad Diego Portales, Santiago, Chile. Disponible en SSRN: https://ssrn.com/abstract=2302809

5. Blanchflower, D. G.y A. Oswald. 2000. The rising well-being of the young. Youth employment and joblessness in advanced countries. University of Chicago Press.

6. $\quad$-a- 2004. "Well-being over time in Britain and the USA". Journal of public economics, 88(7):1359-1386.

7. Cameron, A. C. y P. K. Trivedi. 2005. Microeconometrics: methods and applications. Cambridge University Press.

8. Cruz,J.yJ.Torres. 2006. “De qué depende la satisfacción subjetiva de los colombianos?”. Cuadernos de economía, 25(45):131-154.

9. Di Tella, R. y R. MacCulloch. 2008. "Gross national happiness as an answer to the Easterlin paradox?”. Journal of Development Economics, 86(1):22-42.

10. Di Tella, R., R. J. MacCulloch y A. J. Oswald. 2001. "Preferences over inflation and unemployment: Evidence from surveys of happiness". The American economic review, 91(1):335-341.

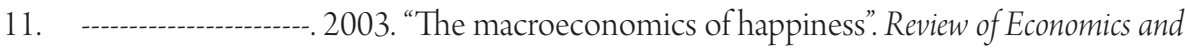
Statistics, 85(4):809-827.

12. Diener, E., R. E. Lucas, S. Oishi y E. M. Suh. 2002. "Looking up and looking down: Weighting good and bad information in life satisfaction judgments". Personality and Social Psychology Bulletin, 28(4):437-445.

13. Dolan, P., T. Peasgood y M. White. 2008. "Do we really know what makes us happy? A review of the economic literature on the factors associated with subjective well-being". Journal of economic psychology, 29(1):94-122. 
14. Easterlin, R. A. 1974. "Does economic growth improve the human lot? Some empirical evidence". Nations and households in economic growth, 89:89-125.

15. Ferrer-i-Carbonell, A. yJ. M. Gowdy. 2007. "Environmental degradation and happiness". Ecological Economics, 60(3):509-516.

16. Frey, B. S. y A. Stutzer. 2000. "Happiness, economy and institutions". The Economic Journal, 110(466):918-938.

17. - --- 2002a. "What can economists learn from happiness research?". Journal of Economic literature, 40(2):402-435.

18. Frey, B. S., A. Stutzer et al. 2002b. "The economics of happiness". World Economics, 3(1):1-17.

19. Galay, K. 2007. "Patterns of time use and happiness in Bhutan: Is there a link between the two". Visiting Research Fellows Series, (432).

20. Graham, C., A. Eggersy S. Sukhtankar. 2004. "Does happiness pay? An exploration based on panel data from Russia”. Journal of Economic Behavior \& Organization, 55(3):319-342.

21. Helliwell, J. F. 2003. "How's life? Combining individual and national variables to explain subjective well-being". Economic Modelling, 20(2):331-360.

22. Lelkes, O. 2006. "Knowing what is good for you: Empirical analysis of personal preferences and the 'objective good". The Journal of Socio-Economics, 35(2):285-307.

23. Louis, V. V. y S. Zhao. 2002. "Effects of family structure, family SES, and adulthood experiences on life satisfaction”. Journal of Family Issues, 23(8):986-1005.

24. Luttmer, E. F. et al. 2005. Neighbors as negatives: Relative earnings and wellbeing. The Quarterly Journal of Economics, 120(3):963-1002.

25. Marks, G. N. y N. Fleming. 1999. "Influences and Consequences of Wellbeing among Australian Young People: 1980-1995”. Social Indicators Research, 46(3):301-323.

26. Medina, C. y J. A. Tamayo. 2012. "An assessment of how urban crime and victimization affects life satisfaction”. Social Indicators Research, (46) 91-147. En: Subjective Well-Being and Security, Springer.

27. Rojas, M. 2009. "Economía de la felicidad. Hallazgos relevantes respecto al ingreso y el bienestar”. El trimestre económico, 76(303):537-573. 
28. Schyns, P. 2001. "Income and satisfaction in Russia". Journal of Happiness Studies, 2(2):173-204.

29. Singer, M. M. 2013. "Bribery diminishes life satisfaction in the Americas". Americas Barometer Insights, 89:1-9.

30. Staubli, S., M. Killias y B. S. Frey. 2014. "Happiness and victimization: an empirical study or Switzerland". European Journal of Criminology, 11(1):57-72.

31. Stutzer, A. 2004. "The role of income aspirations in individual happiness". Journal of Economic Behavior \& Organization, 54(1):89-109.

32. Theodossiou, I. 1998. "The effects of low-pay and unemployment on psychological well-being: a logistic regression approach". Journal of health economics, 17(1):85-104.

33. Thoits, P. A. y L. N. Hewitt. 2001. "Volunteer work and well-being”. Journal of health and social behavior, 42(2), 115-131.

34. Veenhoven, R. 1994. "El estudio de la satisfacción con la vida". Intervención psicosocial, 3:87-116.

35. Victoria García-Viniegras, C. R. e I. González Benítez. 2000. "La categoría bienestar psicológico: su relación con otras categorías sociales”. Revista cubana de medicina general integral, 16(6):586-592.

36. Zurick, D. 2006. "Gross national happiness and environmental status in Bhutan". Geographical Review, 96(4):657-681. 


\section{Anexo 1: Ajuste del modelo}

\begin{tabular}{|c|c|c|c|}
\hline \multicolumn{4}{|c|}{$\begin{array}{c}\text { Cuadro } 1 \\
\text { Clasificación del modelo }(\text { Cutoff }=\mathbf{0 . 7 4})\end{array}$} \\
\hline \multirow[b]{2}{*}{ Classified } & \multicolumn{3}{|c|}{ Test Indicator } \\
\hline & D & $\sim D$ & Total \\
\hline+ & 6409 & 1037 & 7446 \\
\hline- & 3039 & 2090 & 5129 \\
\hline Total & 9448 & 3127 & 12575 \\
\hline \multicolumn{4}{|c|}{ D está definido como qofl = 1 y $\sim$ D representa qofl = 0 . } \\
\hline Sensitivity $\operatorname{Pr}(+1$ & & & $67.83 \%$ \\
\hline Specificity $\operatorname{Pr}(-1$ & & & $66.84 \%$ \\
\hline Positive predictiv & $1+)$ & & $86.07 \%$ \\
\hline Negative predic & $\sim D \mid-)$ & & $40.75 \%$ \\
\hline Correctly classifi & & & $67.59 \%$ \\
\hline
\end{tabular}

Del cuadro anterior es importante analizar dos resultados: la sensibilidad y la especificidad. La sensibilidad muestra la proporción de hogares satisfechos que fueron correctamente clasificados; en este caso, dicha proporción es del 67.83\%. La especificidad es el porcentaje de hogares insatisfechos que fueron correctamente clasificados; este valor es $67.84 \%$. Por otro lado, la exactitud del modelo es de 67.59\%, que muestra la proporción de hogares clasificados de forma acertada.

Para clasificar un hogar como satisfecho, se elige como criterio que la probabilidad estimada sea mayor o igual al cutoff (o valor de corte) que minimiza el tradeoff entre la especificidad y la sensibilidad; dicho valor es 0.74 (ver Gráficos 1 y 2). 


\section{Gráfico 1: Curva ROC}

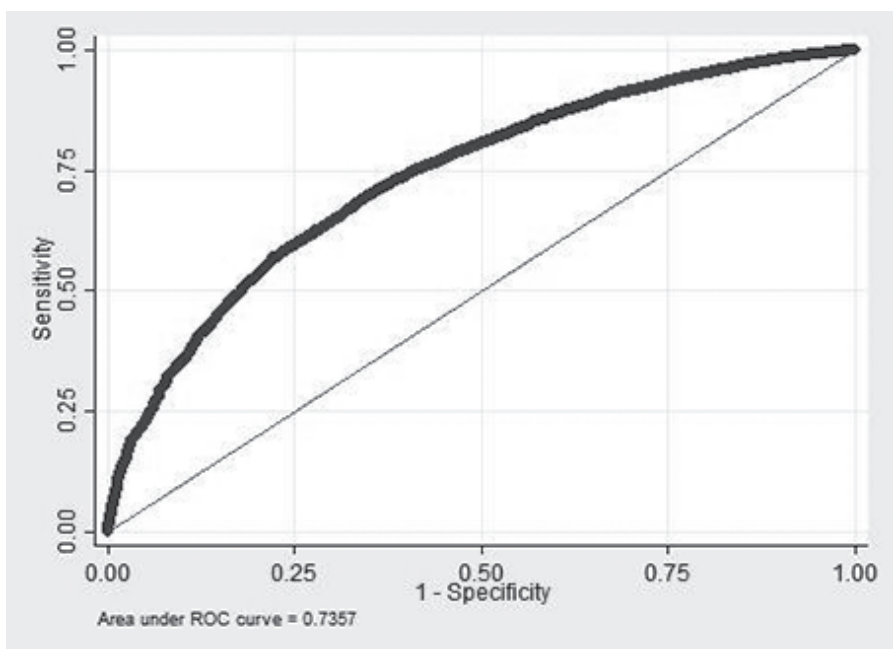

Gráfico 2: Sensibilidad-especificidad y Cutoff

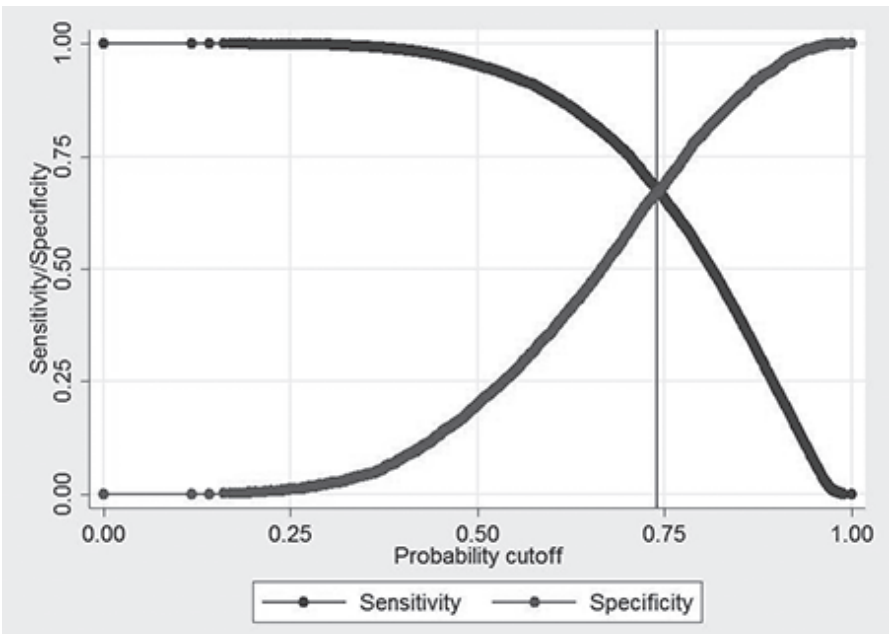




\section{Cuadro 2}

Link Specification Test

\begin{tabular}{lllll}
\hline Logistic regression & Number of obs. & & $=$ & 12575 \\
& LR $x^{2}$ & $=$ & 1701.94 \\
& Prob $>x^{2}$ & & 0.0000 \\
Log likelihood = -6201.9324 & Pseudo $R^{2}$ & & $=$ & 0.1207 \\
\hline \multicolumn{1}{c}{ qofl } & Coeficiente & Error Est. & $\mathbf{z}$ & $\mathbf{P}>\mathbf{z}$ \\
\hline _hat & 1,009465 & 0.055991 & 18.03 & 0.000 \\
_hatsq & $-0,00452$ & 0.023358 & -0.19 & 0.846 \\
_cons & $-0,00189$ & 0.035109 & -0.05 & 0.957 \\
\hline
\end{tabular}

El Cuadro 2 contiene los resultados del link test que permiten identificar problemas de especificación. El concepto inherente a esta prueba es que, si el modelo está correctamente identificado, entonces variables adicionales al modelo no deberían ser estadísticamente significativas, excepto por casualidad. La prueba utiliza el valor lineal estimado (_ hat) y el valor lineal estimado elevado al cuadrado (_hatsq) como regresores para reformular el modelo. La variable _hat debe ser estadísticamente significativa, dado que es el valor estimado del modelo. De otro lado, la variable_hatsq no debe tener mucho poder predictivo; por lo tanto, si esta variable es significativa, se rechaza la hipótesis nula de que el modelo está especificado adecuadamente. En este caso, la variable _hatsq no es significativa; en conclusión, existe evidencia estadística para corroborar que no se omitieron variables relevantes y que la forma funcional es correcta. 


\section{Anexo 2: Definición de las variables}

\begin{tabular}{|c|c|}
\hline Variable & Definición \\
\hline Educación alcanzada & $\begin{array}{l}\text { Variable categórica = } 1 \text { en el nivel de educación alcanzado por el } \\
\text { jefe de hogar. }\end{array}$ \\
\hline Estado civil & Variable categórica = 1 en el estado civil del jefe de hogar. \\
\hline Estrato socioeconómico & $\begin{array}{l}\text { Variable categórica = } 1 \text { en el estrato socioeconómico al que } \\
\text { pertenece la vivienda en la cual reside el hogar. }\end{array}$ \\
\hline Tipo de vivienda & $\begin{array}{l}\text { Variable categórica = } 1 \text { en la categoría del tipo de vivienda en la } \\
\text { cual reside el hogar. }\end{array}$ \\
\hline Seguridad en el barrio & $\begin{array}{l}\text { Variable categórica = } 1 \text { en la categoría de percepción de } \\
\text { seguridad en el barrio donde reside el hogar. }\end{array}$ \\
\hline Ocupación & Variable categórica = 1 en la ocupación del jefe de hogar. \\
\hline Mujer & Variable dicotómica = 1 si el jefe de hogar es mujer. \\
\hline Edad & Variable discreta que contiene la edad del jefe de hogar. \\
\hline Negro & $\begin{array}{l}\text { Variable dicotómica }=1 \mathrm{si} \text { el jefe de hogar es afrodescendiente, }=0 \\
\text { si pertenece a otro grupo étnico. }\end{array}$ \\
\hline Niños en el hogar & $\begin{array}{l}\text { Variable discreta que contiene el número de hijos del jefe de hogar } \\
\text { menores de } 18 \text { años que residen en el hogar. }\end{array}$ \\
\hline Afiliación a Pensiones & $\begin{array}{l}\text { Variable dicotómica = } 1 \text { si el jefe de hogar se encuentra afiliado a } \\
\text { un sistema de pensiones. }\end{array}$ \\
\hline Telefonía & Variable dicotómica = 1 si el hogar posee servicio de telefonía. \\
\hline Gas natural (Red) & $\begin{array}{l}\text { Variable dicotómica = } 1 \text { si el hogar posee servicio de gas natural } \\
\text { por red. }\end{array}$ \\
\hline Gas natural & $\begin{array}{l}\text { Variable dicotómica = } 1 \text { si el hogar posee servicio de gas natural } \\
\text { por pipeta. }\end{array}$ \\
\hline Internet & Variable dicotómica = 1 si el hogar posee servicio de internet. \\
\hline TV por suscripción & $\begin{array}{l}\text { Variable dicotómica = } 1 \text { si el hogar posee servicio de tv por } \\
\text { suscripción. }\end{array}$ \\
\hline Ingreso familiar $\dagger$ & $\begin{array}{l}\text { Variable continua que contiene la sumatoria del ingreso de todos } \\
\text { los miembros del hogar. }\end{array}$ \\
\hline
\end{tabular}

